Post-print version

Copyright: American Society of Civil Engineers

Source: http://ascelibrary.org/doi/full/10.1061/(ASCE)WR.1943-5452.0000592

\title{
Leak Detection and Localization through Demand Components Calibration
}

\author{
Gerard Sanz ${ }^{1}$, Ramon Pérez ${ }^{2}$, Zoran Kapelan ${ }^{3}$ and Dragan Savic ${ }^{4}$
}

\section{ABSTRACT}

The success in the application of any model-based methodology (e.g. design, control, supervision) highly depends on the availability of a well calibrated model. The calibration in water distribution networks needs to be performed online due to the continuous evolution of demands. During the calibration process, background leakages or bursts can be unintentionally incorporated to the demand model and treated as a system evolution (change in demands). This work proposes a leak detection and localization approach to be coupled with a calibration methodology that identifies geographically distributed parameters. The approach proposed consists in comparing the calibrated parameters with their historical values to assess if changes in these parameters are caused by a system evolution or by the effect of leakage. The geographical distribution allows to associate an unexpected behavior of the calibrated parameters (e.g. abrupt changes, trends, etc.) to a specific zone in the network. The performance of the methodology proposed is tested on a real water distribution network using synthetic data. Tested scenarios include leaks occurring at different locations and ranging from $2.5 \%$ to $13 \%$ of the total consumption. Leakage is represented as pressure-dependent demand simulated as emitter flows at the network nodes. Results show that even considering a low number of sensors, leaks with an effect on parameters higher than the parameters' uncertainty can be correctly detected and located within 200 meters.

Keywords: Water Distribution Networks, Leak Detection and Localization, Calibration, Demands.

\section{INTRODUCTION}

Waste and loss of water have been sometimes disregarded due to the low water

\footnotetext{
${ }^{1} \mathrm{PhD}$ student in Dept. of Automatic Control, Polytechnic University of Catalonia, Terrassa 08222, Spain. E-mail: Gerard.Sanz@upc.edu

${ }^{2}$ Associate Professor in Dept. of Automatic Control, Polytechnic University of Catalonia, Terrassa 08222, Spain. E-mail: Ramon.Perez@upc.edu

${ }^{3}$ Professor in College of Engineering, Mathematics and Physical Sciences, University of Exeter, Exeter EX4 4QF, U.K. E-mail: Z.Kapelan@exeter.ac.uk

${ }^{4}$ Professor in College of Engineering, Mathematics and Physical Sciences, University of Exeter, Exeter EX4 4QF, U.K. E-mail: D.Savic@exeter.ac.uk
} 
price and ease of exploitation in developed countries. However, both users and utilities are increasing their concern to avoid present and future water scarcity. Individual users can optimize their daily routines to reduce water waste, but burst and background leakage will be present independently of it.

Leakage in water distribution systems has attracted a lot of attention by both practitioners and researchers over the past years. (Puust et al. 2010) provides a review of leakage management related methods in distribution pipe systems from detection and assessment to efficient control. Leakage identification is divided into leakage awareness and leakage localization (Puust et al. 2010). Leakage awareness focuses on leakage detection in the network [(Kapelan et al. 2003); (Mounce et al. 2010); (Mounce et al. 2011); (Palau et al. 2012); (Romano et al. 2014)], but does not give any information about its precise location. On the other hand, leakage localization (Romano et al. 2013) is an activity that identifies and prioritizes the areas of leakage to make pinpointing of leaks easier. Leak localization techniques can be divided into two categories: external and internal (ADEC 2000). The use of external methods like acoustic logging (Pilcher 2007), penetrating radar (Hugenschmidt and Kalogeropoulos 2009) or liquid detection methods (Henault et al. 2010) has some drawbacks like needing a large number of sensors, not being suitable for application in large urban areas, or being invasive. Internal methods use continuously monitored data to infer the position of leaks using models. Many techniques can be found in literature [(Liggett and Chen 1994); (Vítkovský et al. 2000); (Kim 2005); (Colombo et al. 2009)]. All of these techniques are based on transient analysis, which is mainly used on single, grounded pipelines due to the high effect of the system uncertainty on results. Non-transient modelbased leakage localization techniques have been also developed during the last years [(Wu and Sage 2006); (Pérez et al. 2011); (Wu et al. 2010); (Farley et al. 2011); (Goulet et al. 2013); (Pérez et al. 2014)]. These techniques analyze the difference between measurements and estimated values from leaky scenarios to signal the probability of a zone to contain leakage. Some of these model-based methodologies assume the hypothesis of a single leak in the network [(Goulet et al. 2013); (Pérez et al. 2014)]. Wu et al. (2010) calibrated leakage as a pressure driven demand using the competent genetic algorithm, providing a tool for assisting leakage detection engineers to predict leakage hotspots. Walski et al. (2014) provide some practical suggestions to help users collect the right quality and quantity of data and interpret the results when running genetic algorithms to locate leaks and incorrectly closed valves. Wu and Song (2012) have developed an efficient method to effectively locate the known valves and identify not only their status but also the settings.

The use of models for monitoring and supervising water distribution networks (WDN) is a common practice in water companies. A good calibration of these models is required to obtain reliable results when using them (Sumer and Lansey 2009). Savic et al. (2009) thoroughly reviewed the state of the art of the global calibration problem. Generally, the inverse problem has to be solved using field measurements to adjust the network parameters. Least squares (Kang and Lansey 
2011) and evolutionary methods (Maier et al. 2014) are the most used automatic calibration techniques for WDN models.

Once the model is calibrated, the model-based leak detection and localization methodologies reviewed can make use of it. However, these methodologies do not consider the evolution of demands in the real system. This evolution should be taken into account because demands are parameters that change continuously and leakages may be masked with their evolution.

This work presents a leak detection and localization approach coupled with a Least Squares (LS) based calibration method with geographically allocated demand parameters. The main objective is to diagnose if the updates in the demand model during the continuous calibration correspond to the evolution of demands or to leakage. If leakage is detected, the geographical distribution of parameters allows to identify a particular zone of the network where leakage is most likely located. This leakage can be a burst or any event that induce similar abnormal pressure/flow variations at the district metered area (DMA) level.

\section{PROBLEM STATEMENT}

Goulet et al. (2013) assessed that the most important uncertainty sources are demands and model simplifications, but uncertainty also originates from measurement errors, incorrect boundary conditions, inherent model structural errors or unknown status of valves [(Hutton et al. 2014), (Walski et al. 2014)]. The calibration in this work focuses on demands due to their daily variability and continuous evolution depending generally on social and climate factors comparing to the more stable evolution of roughness. Leakage is considered but not calibrated separately. Therefore, changes in demands have to be analyzed to determine the presence of leakage.

Nodes in WDN models represent an aggregation of multiple demands. Each of these demands may be of different type, e.g. domestic, commercial, etc. Users of the same type are usually assumed to consume water in the same (i.e. similar) way, following a certain, usually pre-determined diurnal demand pattern. The consumption of each user is then computed by multiplying the pattern coefficients with the baseline (i.e. average) demand. Once this is done, demands of different type that are associated with a certain network node are aggregated resulting in the total nodal consumption at given point in time.

However, the information on different types of users associated with a given network node and their diurnal patterns and baseline demands is not always available in practice. Quite often, the only information available is the consumption aggregated during a period of time (usually monthly or quarterly). This low temporal resolution information on demands can still be used to compute the base demand of each consumer. The base demand of a node is computed from the sum of the base demands of consumers aggregated in this node. The basic model presented in Eq. 1 uses the nodal base demands, together with the total network consumption metered at the network inputs, to calculate the demand of each node at each sample. 


$$
\mathbf{d}_{i}(t)=\frac{b d_{i}}{\sum_{j=1}^{n_{d}} b d_{j}} \cdot \mathbf{q}_{\mathbf{i n}}(t)
$$

Where $b d_{i}$ is the base demand of node $i, n_{d}$ is the number of nodes in the network, and $q_{i n}(t)$ is the total network consumption metered at sample $t$.

The demand model presented in Eq. 1 cannot explain the daily variation of the relative pressure behavior between two areas in the network. The demand model in Eq. 2 presents a new approach to model demands depending on their geographical location.

$$
\mathbf{d}_{i}(t)=\frac{b d_{i}}{\sum_{j=1}^{n_{d}} b d_{j}} \mathbf{c}_{j \rightarrow i}(t) \cdot \mathbf{q}_{\mathbf{i n}}(t)
$$

Where $\mathbf{c}_{j \rightarrow i}(t)$ is the value of the demand component $j$ associated to node $i$ depending on the node location. Demand components are calibrated demand multipliers that represent the behavior of nodes in a determined geographical zone, avoiding the dependency on information of the user type and diurnal pattern behavior. All nodes in the same area of node $i$ have the same associated demand component. Consequently, all nodes in the same zone will have the same demand behavior, weighted depending on their base demand. This demand model is capable of generating pressure variations in different zones of the network, as it happens in a real situation. However, the assumption that all nodes in the same area behave exactly in the same way is not realistic. For example, a node in the limit of the effect zone of two demand components should probably have a combination of the behavior of the two demand components, instead of only one. To solve that, we can redefine the demand model in Eq. 2 so that the level to which each demand component is associated with each node is given as a membership, which depends on their geographical location. Eq. 3 represents the new demand model:

$$
\mathbf{d}_{i}(t)=\frac{b d_{i}}{\sum_{j=1}^{n_{d}} b d_{j}} \cdot \mathbf{q}_{\mathbf{i n}}(t) \cdot\left(\alpha_{i, 1} \cdot \mathbf{c}_{1}(t)+\alpha_{i, 2} \cdot \mathbf{c}_{2}(t)+\cdots+\alpha_{i, n_{c}} \cdot \mathbf{c}_{n_{c}}(t)\right)
$$

with

$$
\alpha_{i, 1}+\alpha_{i, 2}+\cdots+\alpha_{i, n_{c}}=1 \quad \forall i
$$

Where $\alpha_{i, j}$ is the association of demand component $j$ with node $i$, and $n_{c}$ is the number of demand components. The membership of each node to each demand component depends on the geographical location of the node, and is computed by means of a sensitivity analysis detailed in (Sanz and Pérez 2015). The model in Eq. 3 is capable of generating different behaviors in every demand, while only having to calibrate few $\left(n_{c}\right)$ demand components.

Sanz and Pérez (2015) presents the demand component calibration process using a LS-based procedure. At each sample, demand components values are estimated so that the errors in predicted measurements are minimized. This way of 

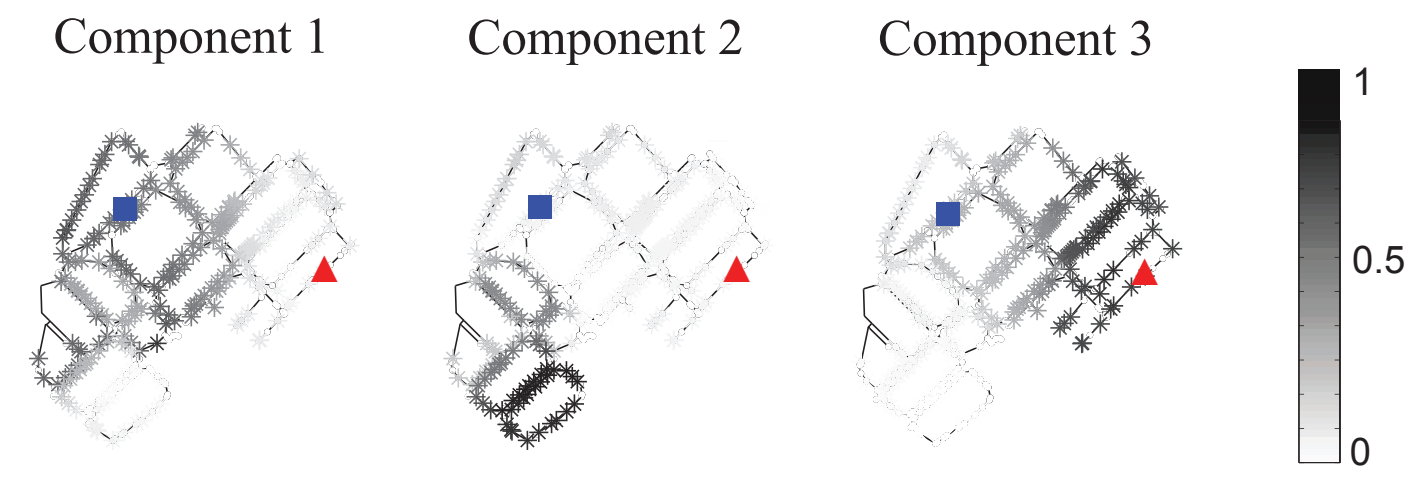

- Node A $\Delta$ Node B

FIG. 1. Example of demand components and memberships in a network

calibrating demands incorporates the usually ignored fact that demands depend in some ways of head status of the network (Giustolisi and Walski 2012). For example, if the pressure in a specific zone of the DMA decreases, the calibration process will estimate demand component values that decrease the consumption of nodes in that zone. Demand components presented in this work should not be confused with the ones in (Giustolisi and Walski 2012), where demand components were generated with a previous knowledge of the use of water (human-based, volume-based, non-controlled orifice-based, leakage-based).

The calibrated demand components generate individual demands that may not be exactly as the real ones, but the aggregated demand in a zone at a specific sample, and the cumulative demand of each individual node during a period of time (similar to the billing) will coincide with the real ones if other parameters (roughness, valve status, etc.) are well calibrated.

Fig. 1 presents a network where three demand components have been defined as explained in (Sanz and Pérez 2015). The first component is located on the North-West side of the DMA; the second component is located on the South-West of the DMA; and the third component is located on the East side of the network. The memberships are depicted in greyscale: the darker the color of a node, the higher the membership of that node to the demand component. Tab. 1 presents the memberships of the two nodes highlighted in Fig. 1. Demand of node A is affected $(60 \%)$ by the value of demand component 1 , while component 3 has a lower $(35 \%)$ effect on it. On the other hand, demand of node B is completely (99\%) affected by demand component 3. Demand component 2 does not have any effect on both demands, as it is far (geographically and hydraulically) from the two example nodes.

A comparison of the calibration results between type of user-based demand patterns and pressure sensitivity-based demand components is presented in (Sanz 


\section{TABLE 1. Memberships of nodes A and B of the example network}

\begin{tabular}{|l|c|c|}
\hline \multicolumn{1}{|c|}{ Node } & $\mathrm{A}$ & $\mathrm{B}$ \\
\hline Membership to $\mathbf{c}_{1}$ & 0.6 & 0.01 \\
\hline Membership to $\mathbf{c}_{2}$ & 0.05 & 0.01 \\
\hline Membership to $\mathbf{c}_{3}$ & 0.35 & 0.99 \\
\hline
\end{tabular}

and Pérez 2014), with better results for the latter: the uncertainty in the calibrated parameters is reduced, while the geographical distribution is useful for applications requiring parameters to be related with zones of the network. Sanz and Pérez (2015) present the methodology to select the sensors that have high sensitivity to one demand component while being low sensitive to the rest.

Not considering leakage estimation in the online calibration process leads to the inclusion of possible losses in the calibrated demand model. Therefore, the key factor is to distinguish whether the evolution of calibrated demands is true or hides leakage. The demand model presented in Eq. 3 allows to detect and locate leaks straightforwardly through calibration due to the geographical distribution of the calibrated parameters.

This work considers the following assumptions:

- A maximum of one leak appears in the network.

- Pressures and flows at the network inputs are known.

- A set of pressures measurements within the DMA is available.

- Noise is considered in the measurements.

- Quarterly billing for each individual consumer is known.

- The methodology is applied to a real network with synthetic data where uncertainty in demands is considered.

- Gross errors in field data and model are considered to be corrected at a prior stage.

- Sudden weather changes or other special events that may produce relevant demand variations are not considered.

- Status of valves in the DMA have been checked as part of the prior calibration process.

\section{METHODOLOGY}

Fig. 2 presents the structure of the coupled calibration and leak detection and localization methodologies. Measurements taken from the real network are introduced via the SCADA system, where a validation process is performed first. The calibration process estimates every hour the set of current demand components $\mathbf{c}^{c}$ that minimize the errors in model predictions. This set of calibrated demand components is stored into a database, where it is concatenated to previous hours and days. Simultaneously, the detection process compares the sets of calibrated and historical demand components. Assuming that consumers' habits do not change significantly from one week to another, a demand component value 


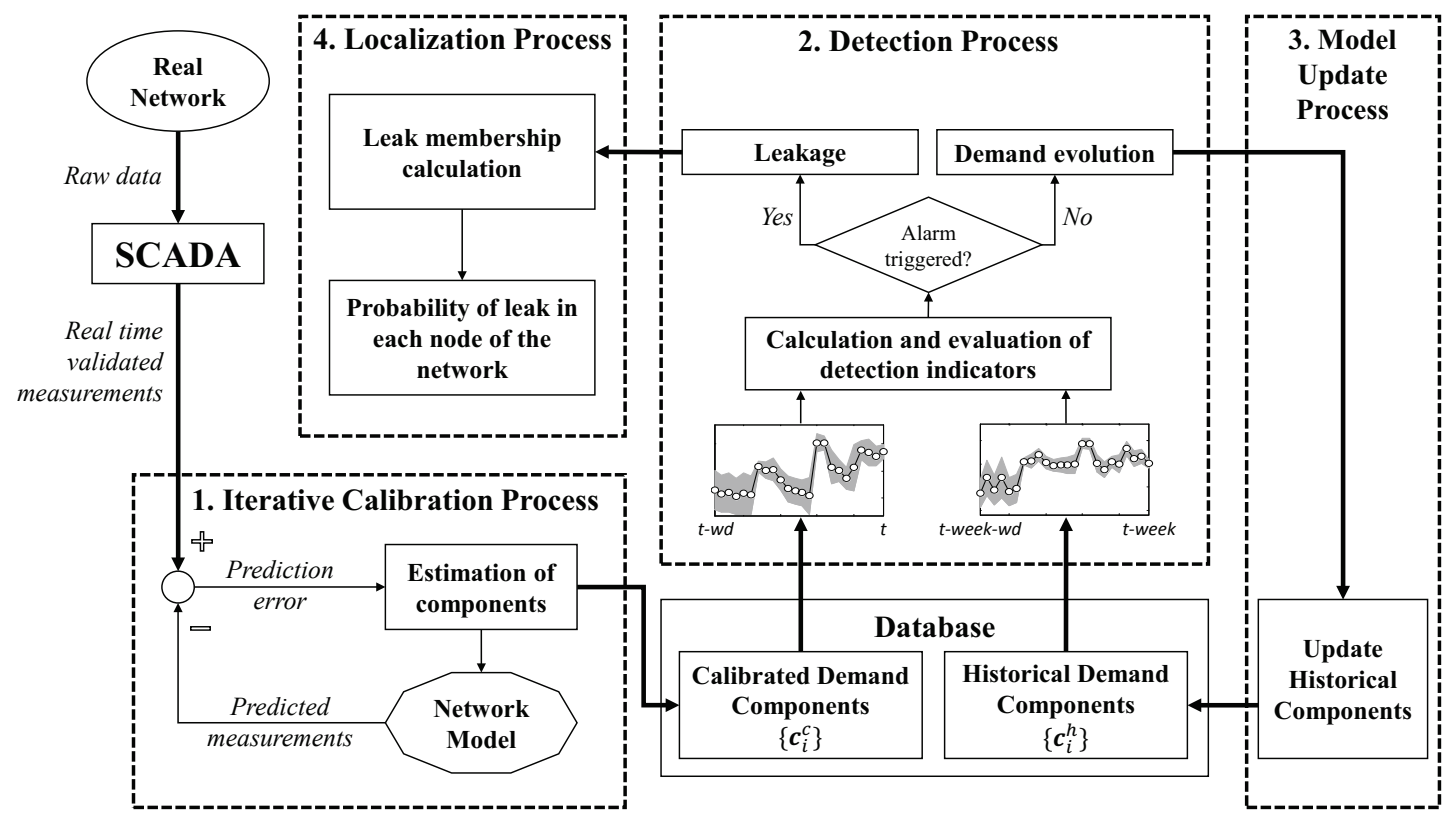

FIG. 2. Scheme of the calibration process coupled with the model update/leakage detection and localization processes

$\mathbf{c}_{i}^{c}$ is expected to be similar to the corresponding value in the previous week $\mathbf{c}_{i}^{h}$ (historical component). At time $t$, the last $w d$ values of each component $\mathbf{c}_{i}^{c}$ are compared with the same time window of $\mathbf{c}_{i}^{h}$ using detection indicators, where $w d$ is the number of samples to be compared (e.g. if $w d=24,24$ hours of $\mathbf{c}_{i}^{c}$ will be compared with the same 24 hours of $\mathbf{c}_{i}^{h}$ ). If detection indicators do not trigger the detection alarm, the state of the network is classified as non-faulty, and the historical demand components values are updated with the currently calibrated ones (model update process). The new demand components include slight changes in demands due to the evolution of the system. On the contrary, if the detection alarm is triggered, the leak localization process starts. The weekto-week comparison is useful not only for the similarity of the compared days, but also to avoid false alarms from progressive changes due to seasonal habits in population.

The calibration process included in Fig. 2 is described in (Sanz and Pérez 2015). The current work focuses on the description of the detection and localization processes.

\section{Detection indicators}

Six detection indicators are defined to evaluate the similarity or dissimilarity between calibrated and historical demand components: Pearson correlation, conditional overlapping, unit norm, relative increment in mean component values and consumption, and relative residual coefficient. A description of each indicator 
is listed next:

- The Pearson correlation is a measure of the linear dependence between the two components $\mathbf{c}_{i}^{c}$ and $\mathbf{c}_{i}^{h}$.

$$
\boldsymbol{\rho}_{i}(t)=\frac{\sum_{k=t-w d+1}^{t}\left[\left(\mathbf{c}_{i}^{c}(k)-\bar{c}_{i}^{c}\right)\left(\mathbf{c}_{i}^{h}(k)-\bar{c}_{i}^{h}\right)\right]}{\sqrt{\sum_{k=t-w d+1}^{t}\left(\mathbf{c}_{i}^{c}(k)-\bar{c}_{i}^{c}\right)^{2} \cdot \sum_{k=t-w d+1}^{t}\left(\mathbf{c}_{i}^{h}(k)-\bar{c}_{i}^{h}\right)^{2}}}
$$

where $\mathbf{c}_{i}^{c}$ comprises times from $t-w d+1$ to $t$, and $\mathbf{c}_{i}^{h}$ comprises the same times but corresponding to the previous week; and $t$ is a specific point in time where calibrated components are available. Correlations close to 1 indicate a high similarity between components.

- The overlapping coefficient measures the overlap between two discrete or continuous probability density functions (pdf).

$$
\mathbf{o}_{i}(k)=\int_{-\infty}^{\infty} \min \left(f_{i}(x), g_{i}(x)\right) d x
$$

where $f_{i}(x)$ is the pdf of the current calibrated component at sample $k$; and $g_{i}(x)$ is the pdf of the historical component at the same sample of the previous week. The mean overlapping $\overline{\mathbf{o}}_{i}$ during a time window is calculated as seen in Eq. 6.

$$
\overline{\mathbf{o}}_{i}(t)=\frac{1}{w d} \sum_{k=t-w d+1}^{t} \mathbf{o}_{i}(k)
$$

A $100 \%$ overlap is obtained with equal probability distributions. As the pdfs become different, the overlapping decreases. A new indicator called conditional overlapping coefficient can be defined considering only the reduction of overlapping coefficients due to positive component changes (increase in consumed water).

$$
\mathbf{c o}_{i}(t)= \begin{cases}\overline{\mathbf{o}}_{i}(t) & \overline{c_{i}^{c}}>\overline{c_{i}^{h}} \\ 100 \% & \text { otherwise }\end{cases}
$$

- Norms are functions that assign a strictly positive length or size to a vector in a vector space, other than the zero vector.

$$
\left\|\mathbf{c}_{i}^{c}-\mathbf{c}_{i}^{h}\right\|_{p}(t)=\sqrt[p]{\sum_{k=t-w d+1}^{w d}\left|\mathbf{c}_{i}^{c}(k)-\mathbf{c}_{i}^{h}(k)\right|^{p}}
$$

Only the unit norm $(p=1)$ is considered. 
- The relative increment in mean component values $\Delta \mathbf{c}_{i}$ indicates the percentage of relative increment between the current values (averaged through a defined time) and the historical ones (also averaged).

$$
\Delta \mathbf{c}_{i}(t)=100 \cdot \frac{\overline{c_{i}^{c}}-\overline{c_{i}^{h}}}{\bar{c}_{i}^{h}}
$$

where the means have been computed during a time interval $w d$.

- The relative increment in mean component consumption $\Delta \mathbf{c}_{i}^{\mathbf{d}}{ }_{i}$ indicates the percentage of relative increment between the current consumption (averaged through a defined time) and the historical one (also averaged). This indicator is similar to the previous one, but the components' consumptions in $1 / \mathrm{s}$ are used instead of the dimensionless values.

$$
\Delta \mathbf{c}^{\mathbf{d}}{ }_{i}(t)=100 \cdot \frac{\sum_{k=t-w d+1}^{w d}\left(\mathbf{c}_{i}^{c}(t) \cdot \mathbf{q}_{\mathbf{i n}}{ }^{c}(t)\right) / t-\sum_{k=t-w d+1}^{w d}\left(\mathbf{c}_{i}^{h}(t) \cdot \mathbf{q}_{\mathbf{i n}}{ }^{h}(t)\right) / t}{\sum_{k=t-w d+1}^{w d}\left(\mathbf{c}_{i}^{c}(t) \cdot \mathbf{q}_{\mathbf{i n}^{c}}{ }^{c}(t)\right) / t}
$$

where superscripts ${ }^{c}$ and ${ }^{h}$ in qin $_{\text {in }}$ refer to current and historical total inflow, respectively.

- The relative residual coefficient gives a measure about the relative variation between two probability distributions considering the $95 \%$ confidence intervals.

$$
\operatorname{rRes}_{i}(t)=\frac{100}{w d} \sum_{k=t-w d+1}^{t} \frac{\left(\mathbf{c}_{i}^{c}(k)-1.96 \sigma_{\mathbf{c}_{i}^{c}}(k)\right)-\left(\mathbf{c}_{i}^{h}(k)+1.96 \sigma_{\mathbf{c}_{i}^{h}}(k)\right)}{\left|\mathbf{c}_{i}^{h}(k)+1.96 \sigma_{\mathbf{c}_{i}^{h}}(k)\right|}
$$

This measure only gives positive values when the current component lower bound is higher than the $95 \%$ upper bound of the historical component.

\section{Setting of thresholds}

The presented detection indicators evaluate the variation in demand components by comparing the current components' values with the previous week ones. As the variations become higher, the probability of having an anomaly in the network increases. Variations in demand components have different effects on detection indicators; e.g. the unit norm is sensitive to changes in the component average value, whereas the conditional overlapping only considers positive changes in it. Therefore, the six indicators are combined to obtain a more robust detection.

Each detection indicator gives a score to each demand component depending on its variation. The sum of scores is then used to decide if the component has an anomaly or not. The scores given by the detection indicators depend on thresholds. The definition of a unique threshold for each indicator may produce poor leakage detection or excessive false alarms. Instead, two thresholds are defined for each indicator, giving 1 or 2 score points when overtaking the first 
and second threshold, respectively. Detection indicators' thresholds are defined separately, but shared by all demand components.

The thresholds values are determined through a training process when no leakage is present in the network. The mean and standard deviation of each detection indicator are computed during the non-faulty scenario. Then, the thresholds are set so that the probability of data being under the low detection threshold is $80 \%$, and the probability of data being under the high detection threshold is $95 \%$, for the worst component in each indicator. The worst case is used to avoid false alarms. Finally, the global threshold (sum of individual scores) is set so that the total sum of the non-faulty indicators is under this value. The thresholds setting proposed is performed in a way that if the network remains in the same state, the probability of data falling outside thresholds is $20 \%$ for the lower detection threshold and $5 \%$ for the higher one, for the worst component in each indicator.

In the end, we have a system that triggers the alarm in a particular demand component if the total score for that component is higher than the global threshold. As a result, the methodology is able not only to detect the leakage, but also to classify it in a determined demand component, which is associated to a specific zone of the network.

\section{Effect of undetected anomalies}

Setting the thresholds for the leak detection and localization process is assumed to be done over a non-faulty state of the network. However, different types of errors or anomalies can exist both in the model or network, like undetected bursts, existing background leakages, unknown status valves (Walski et al. 2014), or bad estimated roughness, among others. The presence of these anomalies can be treated depending on when the anomaly has appeared without being aware of it:

1. Before setting the thresholds: The undetected anomaly will hinder the best demand adjustment. Nevertheless, this anomaly will be incorporated into the calibrated demand components model. Consequently, the methodology will be able to detect new bursts that cause a change in the components from that moment on.

2. After setting the thresholds: The currently calibrated demand components will accommodate their values to adapt to the new network pressures, provoking a change compared to the historic demand components. Future studies will analyze this scenario to observe if the methodology is able to detect and locate the non-burst anomalies. These events may induce similar pressure-flow variations in the network as the ones produced by bursts.

This work assumes that none of this anomalies are present before or after the setting of the thresholds. 


\section{Localization}

This section presents two methods (direct method and leak membership method) to interpret the geographical information contained in the nodes' memberships and locate the detected leak.

The direct method locates the leak depending on the membership of each node to the abnormal demand component. The higher the membership of a node to the abnormal component, the higher the probability of leak occurring in that node. The geographical distribution of demand components will indicate a particular zone in the network with high probability to contain the leak.

The leak membership method consists in calculating the theoretical leak memberships to demand components. When leakage is present, pressures decrease due to the increasing flow. Consequently, the calibration process modifies the demand components values to adapt the model to the new pressures. Therefore, all components suffer higher or lower variations that can be attributed to the leak. These variations define the theoretical leak memberships. Subsequently, the leak memberships are compared with the ones from all network nodes using the Pearson correlation. The higher the correlation in a node, the higher the probability of that node to contain the leak.

\section{CASE STUDY}

The leak detection and localization methodology is applied to a real network model with synthetic data. The network is a DMA situated in the Barcelona neighborhood of Nova Icaria. It is composed of 3455 pipes and 3377 junctions, as depicted in Fig. 3. Water is supplied to the network through two pressure reduction valves, highlighted in Fig. 3 with a triangle and a circle. Pressure and flow are monitored at both water inlets with a sample time of 10 minutes. The resolution is $0.01 \mathrm{l} / \mathrm{s}$ for the flow sensors, and $0.01 \mathrm{mwc}$ (meters of water column) for both the inlet and pressure sensors within the DMA. Although high resolution data cannot be directly provided by real sensors, this could be achieved by oversampling (Pandya and Gupta 2014), which is also useful to filter noise. Status of all valves in the network is known. The mean daily consumption is of about $33 \mathrm{l} / \mathrm{s}$, with a minimum night flow of $20 \mathrm{l} / \mathrm{s}$ and peak hour flows of $50 \mathrm{l} / \mathrm{s}$.

\section{Synthetic data generation}

The generation of synthetic data requires a previous emulation of reality. A complete set of synthetic demands has been computed to represent reality, where different consumers use water differently (e.g. household, commercial, industrial, etc.). First, ten diurnal demand patterns have been defined, representing different types of users. Each nodal demand in the network has an associated type of user. These types are mixed all over the network, emulating the real behavior of the used DMA. All patterns, and consequently all nodal demands, have different behaviors during weekdays and weekends. A random normal noise $N\left(0,0.1 \cdot \mathbf{d}_{i}(t)\right)$ has been added to each individual demand at each sample, where $\mathbf{d}_{i}(t)$ is the consumption of node $i$ at sample $t$ without noise. 


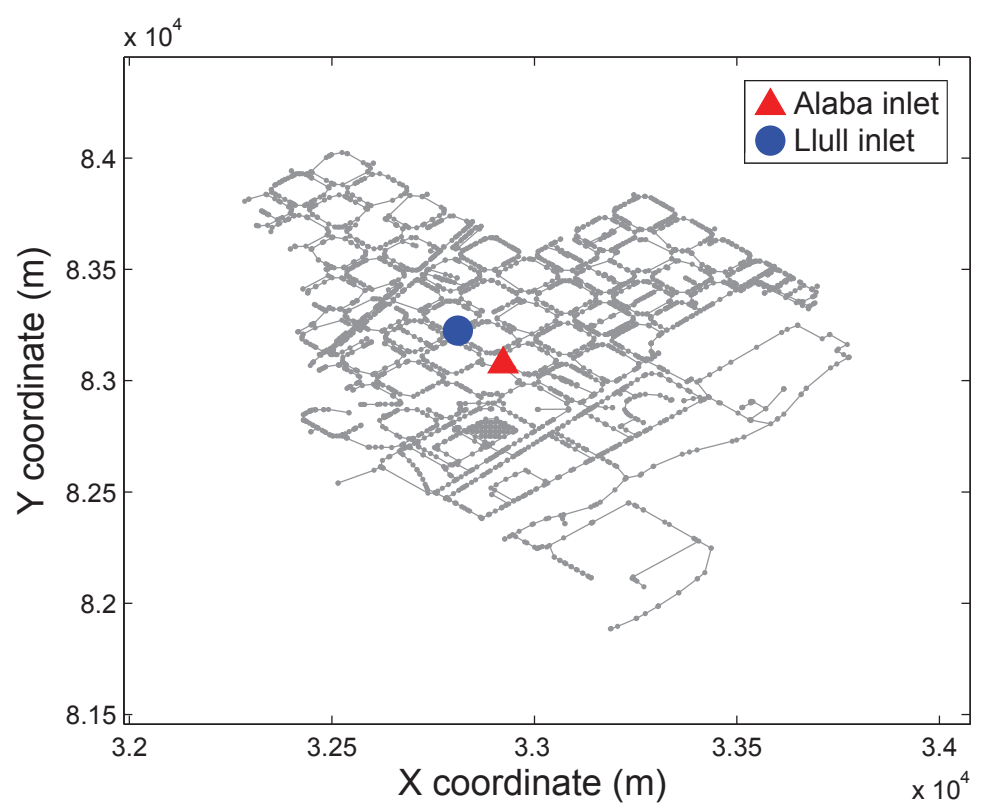

\section{FIG. 3. Nova Icaria DMA EPANET model with highlighted inputs}

Finally, the network model is simulated using EPANET in order to obtain pressures at the defined sensors and distribution of flows at the inputs. Base demands and boundary conditions (total flow and pressure set points) have been obtained from real measurements provided by the Barcelona water utility AGBAR. A random noise $\mathrm{N}(0,0.01 \mathrm{mwc})$ has been added to pressure measurements after simulating the network.

\section{Calibration parameters}

The number of demand components and sensors used depends on both the final application of the calibration and the budget for installing sensors. This work considers a small number of sensors (five) in order to mimic a situation typically found in the real network, where a small number of (e.g five) pressure sensors will be installed by the water company. These five sensors restrict the number of demand components that can be calibrated, as the system of equations in the well formulated calibration problem has to be over or equally determined. Consequently, the methodology presented in the problem statement section will be used to define the memberships of nodes to five demand components, and the location of the five pressure sensors that are going to be used. Flow sensors will be considered in future studies.

Fig. 4 depicts the distribution of demand components (greyscale maps) and sensors (circles). The geographical distribution of demand components can be observed through the nodes memberships: the higher the membership, the darker the color in Fig. 4. 


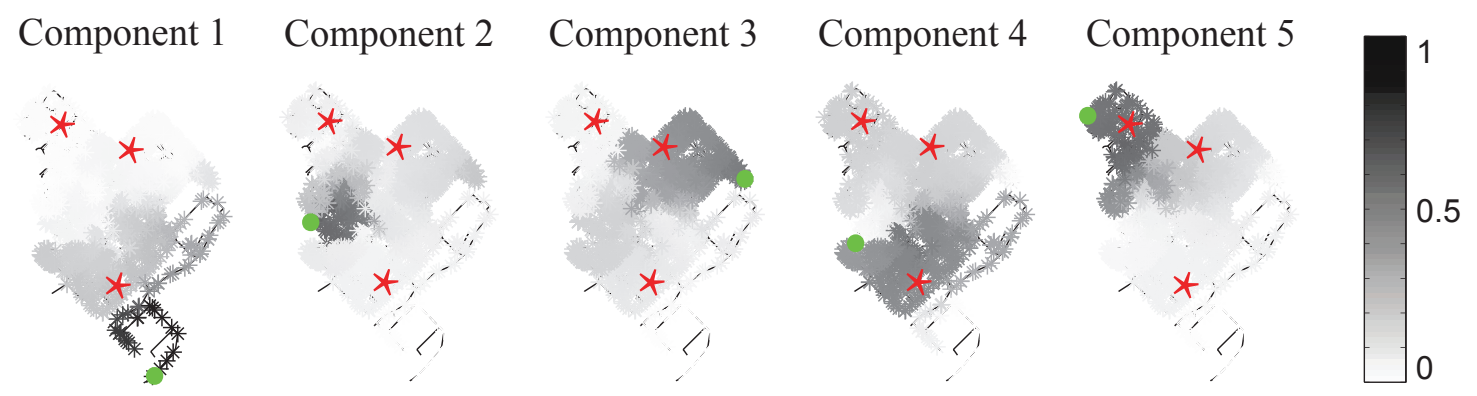

FIG. 4. Greyscale maps of nodes memberships to each demand component, sensors (circles) and simulated leaks (stars)

TABLE 2. Summary of the generated leakage scenarios

\begin{tabular}{|c|c|c|c|c|c|c|c|c|c|}
\hline Leak & \multicolumn{3}{|c|}{ L1 } & \multicolumn{3}{c|}{ L2 } & \multicolumn{3}{c|}{ L3 } \\
\hline Scenario & $\mathrm{S} 1$ & $\mathrm{~S} 2$ & $\mathrm{~S} 3$ & $\mathrm{~S} 4$ & $\mathrm{~S} 5$ & $\mathrm{~S} 6$ & $\mathrm{~S} 7$ & $\mathrm{~S} 8$ & $\mathrm{~S} 9$ \\
\hline $\begin{array}{c}\text { Mean daily } \\
\text { water } \\
\text { discharge }\end{array}$ & $5 \mathrm{l} / \mathrm{s}$ & $3 \mathrm{l} / \mathrm{s}$ & $1 \mathrm{l} / \mathrm{s}$ & $5 \mathrm{l} / \mathrm{s}$ & $3 \mathrm{l} / \mathrm{s}$ & $1 \mathrm{l} / \mathrm{s}$ & $5 \mathrm{l} / \mathrm{s}$ & $3 \mathrm{l} / \mathrm{s}$ & $1 \mathrm{l} / \mathrm{s}$ \\
\hline $\begin{array}{c}\text { \% of total } \\
\text { consumption }\end{array}$ & $13 \%$ & $8 \%$ & $2.5 \%$ & $13 \%$ & $8 \%$ & $2.5 \%$ & $13 \%$ & $8 \%$ & $2.5 \%$ \\
\hline
\end{tabular}

\section{Generation of scenarios}

Nine leakage scenarios have been generated to evaluate the performance of the methodology developed. Leaks are assumed to be located at the nodes of the network. This simplification implies a loss of accuracy of the order of the pipe length. Such simplification can be assumed if the maximum localization error required by the company is greater than this length (Pérez et al. 2014). In order to simulate a leak, an emitter coefficient $C_{e}$ is set in a node so that the leak size generated depends on the pressure of that node (Rossman 2000), as described in Eq. 12.

$$
q=C_{e} \cdot p^{\gamma}
$$

where $q$ is the leak water discharge; $C_{e}$ is the emitter coefficient; $p$ is the pressure at the node; and $\gamma$ is an exponent of about 0.5 (Hazen-Williams, Darcy-Weisbach, Chezy-Manning formulas (Rossman 2000)).

Three different locations (signaled in Fig. 4 with stars) and three different sizes of leaks are tested. Leak 1 (L1) is located in the effect zone of component $\mathbf{c}_{5}$; leak 2 (L2) is located in the effect zone of component $\mathbf{c}_{3}$; and leak 3 (L3) is located in the effect zone of component $\mathbf{c}_{4}$. Tab. 2 presents the main characteristics of the generated scenarios.

Results presented in the following section consider leaks appearing at low consumption hours. Additional scenarios (not included in this work) where leaks 


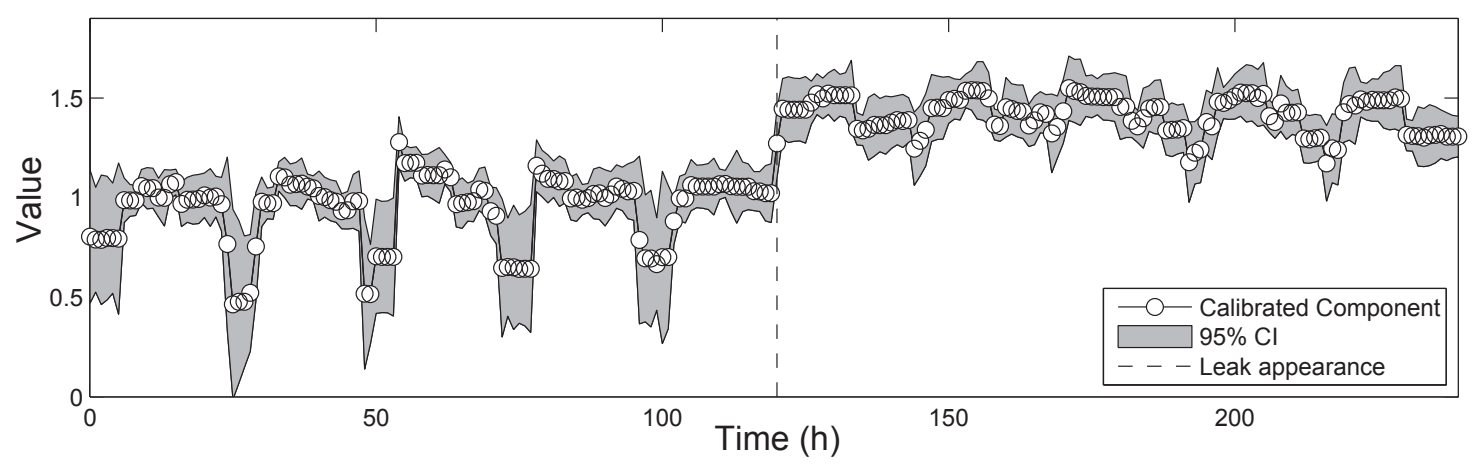

FIG. 5. Calibrated component $c_{5}$ during a non-faulty week and a faulty week with a $5 \mathrm{l} / \mathrm{s}$ leakage

occur at the peak consumption hour have been also tested, obtaining similar results.

\section{RESULTS}

This section presents the results when applying the methodology combining calibration, leak detection and localization.

\section{Calibration}

The calibration process is applied considering the five components and sensors that have been selected in the previous section. As mentioned by Walski et al. (2014), it is necessary to have head loss in the system that is significantly greater than the error in measurement to avoid random adjustments. In the current case study, the maximum head loss is of about $7.2 \mathrm{~m}$, which fulfills the mentioned requirement. The values of the five demand components are calibrated by minimizing the error in pressure and flow measurements at each hour using the LS-based methodology detailed in (Sanz and Pérez 2015). The uncertainty calculation is done by propagating the sensors' noise using the First Order Second Moment model (Lansey et al. 2001). Fig. 5 depicts two weeks (without weekends) of calibrated component $\mathbf{c}_{5}$ and its $95 \%$ confidence intervals. The first week (day 1 to 5) represents a non-faulty scenario. At the beginning of the second week (days 6 to 10), a $5 \mathrm{l} / \mathrm{s}$ leakage appears.

The validation of the calibrated components is done by comparing the proportion of consumed water calculated from the calibrated values with the one calculated from billing. Fig. 6 depicts this validation in two scenarios: a) No leakage scenario; and b) $5 \mathrm{l} / \mathrm{s}$ leakage scenario. Each of the radius represents a different demand component. Fig. 6.a verifies the success of the calibration, whereas Fig. 6.b warns of a bad calibration that has to be analyzed.

\section{Selection of detection indicators' time windows and thresholds}

The six detection indicators presented in the methodology section have to be detailed for the current case study. A time window of $12 \mathrm{~h}$ is selected for the 
a)

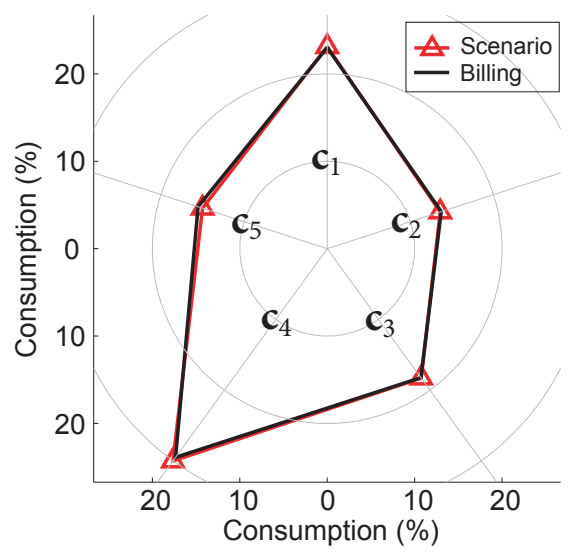

b)

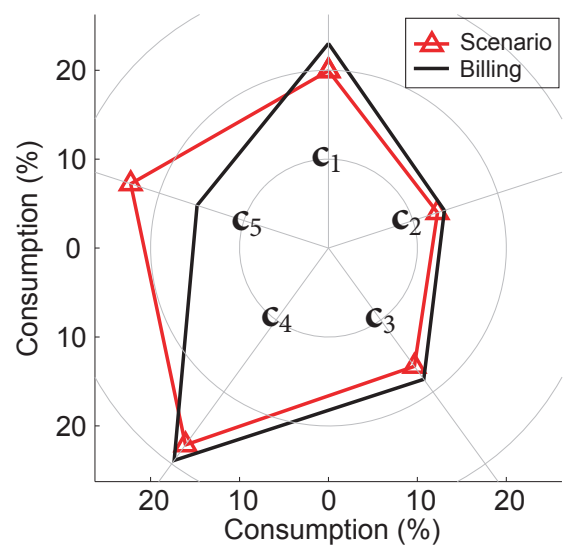

FIG. 6. Percentage of demand components consumption from billing and calibrated components: a) Scenario with no leakage; and b) Scenario with $5 \mathrm{l} / \mathrm{s}$ leakage in component 5

calculation of the detection indicators to detect changes in a fast but reliable way. However, the correlation and unit norms indicators have to be computed with a $24 \mathrm{~h}$ time window due to their instability when calculated with a narrower window.

The selection of thresholds has to be done on a non-faulty state of the network. In this work, the non-faulty scenario is known (Fig. 6.a). In a real case, the validation of the calibration presented in Fig. 6 would be used to advise about the state of the network. In case of network experiencing undetectable burst or background leakage (Fig. 6.b) before applying the methodology presented, this leakage would be considered as part of the demand model and thresholds would be set without taking it into account. The methodology would still be able to detect and locate new leaks occurring from that moment on.

Fig. 7 shows the six indicators with the defined thresholds for each one. The $80 \%$ and $95 \%$ confidence intervals (CI) are marked with dashed and dash-dotted lines, respectively. These thresholds have been computed using the component with highest probability of having a false alarm during the non-faulty scenario in each of the detection indicators.

Fig. 8 depicts the sum of scores obtained from the indicators. Only demand components $\mathbf{c}_{1}, \mathbf{c}_{2}$ and $\mathbf{c}_{5}$ get no null scores during the non-faulty scenario. The highest score is obtained in demand component $\mathbf{c}_{1}$ with a value of 3 . Consequently, the global detection threshold is set at a value of 4 (dashed line in Fig. 8).

\section{Leak detection and localization}

The methodology is tested using the nine faulty scenarios defined in Tab. 2 plus a non faulty scenario (S0). Tab. 3 sums up the results for all the scenar- 

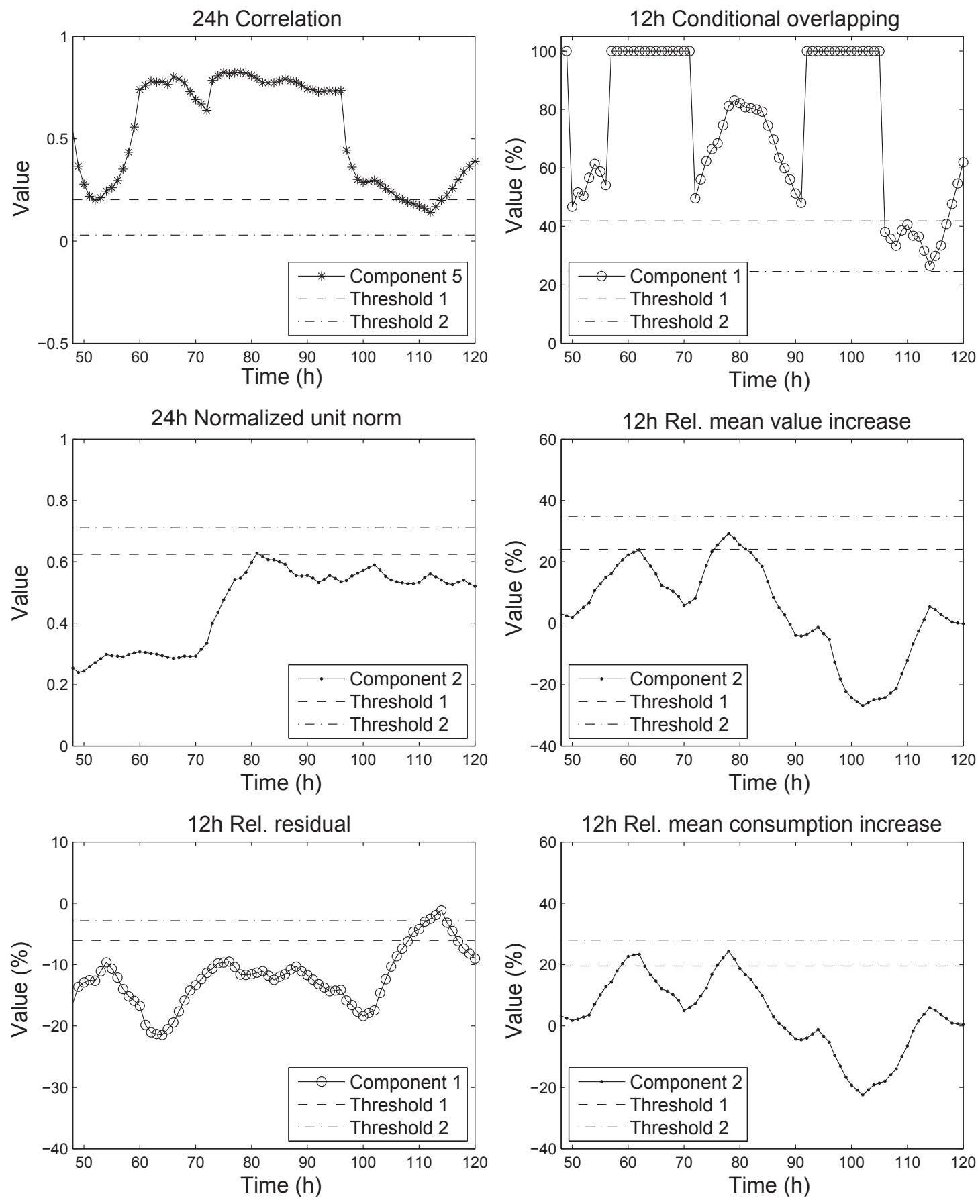

FIG. 7. Detection indicators during the non-faulty scenario with defined thresholds in dashed and dash-dotted lines 


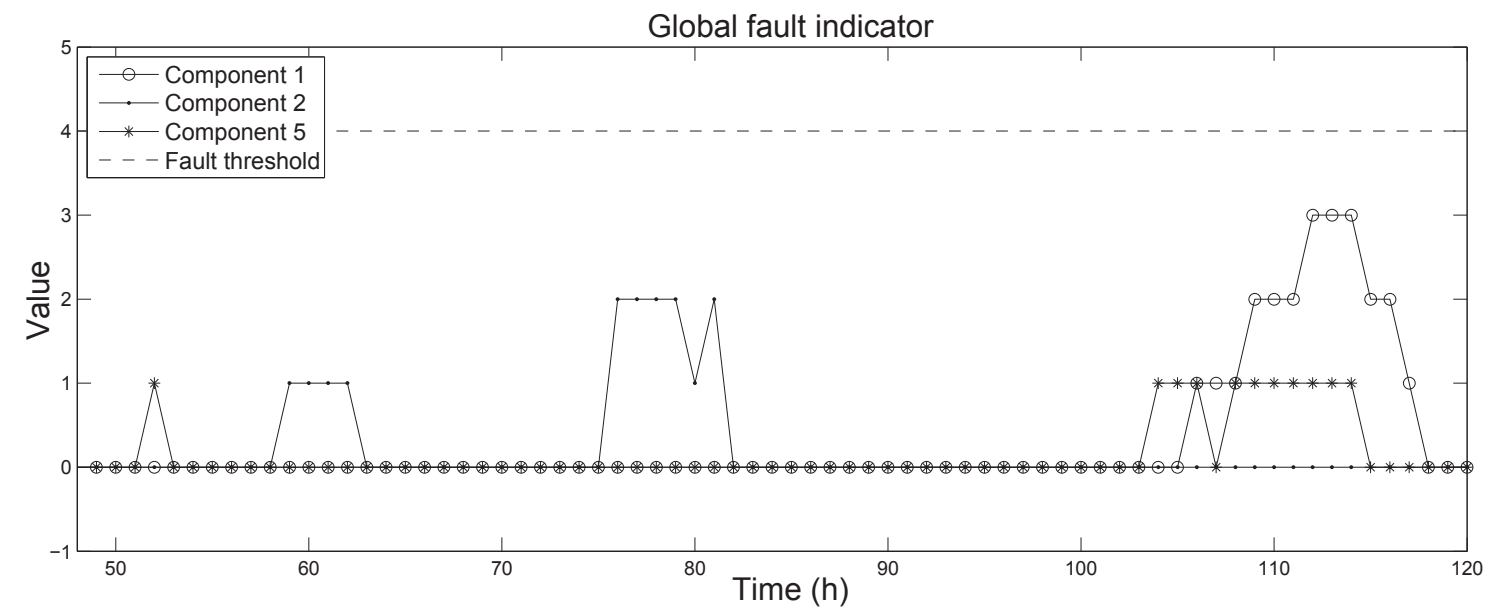

FIG. 8. Total detection score for all components with global detection threshold set at a value of 4

TABLE 3. Summary of results for each scenario

\begin{tabular}{|c|c|c|c|c|c|c|c|c|c|c|}
\hline Scenario & S0 & S1 & S2 & S3 & S4 & S5 & S6 & S7 & S8 & S9 \\
\hline Leak detected & - & Yes & Yes & Yes & Yes & Yes & Yes & Yes & Yes & No \\
\hline Detection time & - & $3 \mathrm{~h}$ & $4 \mathrm{~h}$ & $4 \mathrm{~h}$ & $4 \mathrm{~h}$ & $6 \mathrm{~h}$ & $6 \mathrm{~h}$ & $6 \mathrm{~h}$ & $10 \mathrm{~h}$ & - \\
\hline $\begin{array}{c}\text { Geogr. distance } \\
\text { to real leak } \\
\text { [direct] (m) }\end{array}$ & - & $\mathbf{1 8 3}$ & 183 & $\mathbf{1 8 3}$ & 657 & 657 & 657 & 220 & 220 & - \\
\hline $\begin{array}{c}\text { Geogr. distance } \\
\text { to real leak [leak } \\
\text { memb.] (m) }\end{array}$ & - & 224 & $\mathbf{1 7 7}$ & $\mathbf{1 8 3}$ & $\mathbf{2 0 6}$ & $\mathbf{1 8 5}$ & $\mathbf{5 2 7}$ & $\mathbf{1 4 5}$ & $\mathbf{1 4 5}$ & - \\
\hline $\begin{array}{c}\text { Pipe distance to } \\
\text { real leak [direct] } \\
\text { (m) }\end{array}$ & - & $\mathbf{2 3 1}$ & $\mathbf{2 3 1}$ & $\mathbf{2 3 1}$ & 857 & 857 & 857 & 365 & 365 & - \\
\hline $\begin{array}{c}\text { Pipe distance to } \\
\text { real leak [leak } \\
\text { memb.] (m) }\end{array}$ & - & 396 & $\mathbf{2 3 1}$ & $\mathbf{2 3 1}$ & $\mathbf{2 9 3}$ & $\mathbf{2 6 3}$ & $\mathbf{6 9 8}$ & $\mathbf{1 8 1}$ & $\mathbf{1 8 1}$ & - \\
\hline
\end{tabular}

ios in terms of detection, detection time and localization accuracy. Accuracy is presented as the distance (geographic and pipe distance) between the real leak and the node selected by the methodology as the one with highest probability to contain the leak. These distances are computed for both the direct method and the leak membership method. The best result for each distance is highlighted in boldface letter.

Fig. 9 depicts the graphical results for scenarios S3 (Fig. 9.a,b), S4 (Fig. 9.c,d) and S8 (Fig. 9.e,f) using greyscale maps. The first column of subfigures (Fig. 9.a,c,e) 
refers to the direct method, whereas the second column (Fig. 9.b,d,f) refers to the leak membership method. The darker the color in the greyscale map, the higher probability of the node to contain the leak.

Fig. 10 depicts the geographical distance of all nodes in the network from the real leak (x axis), together with the indicator that gives a probability for the fault occurring in each node (y axis). For the direct approach (Fig. 10.a,c,e), the indicator is the normalized membership; and for the leak membership approach (Fig. 10.b,d,f), the indicator is the correlation. Each row of subfigures corresponds to scenarios S3 (Fig. 10.a,b), S4 (Fig. 10.c,d) and S8 (Fig. 10.e,f). The node with the highest indicator value is shown with a dashed line. Fig. 11 depicts the same information but this time in terms of pipe distance from each node to the real leak. This distance helps to assess the use of acoustic methods that can locate precisely the leak if it is within a determined pipe distance. The teams looking for the leak would start from the node with highest probability of containing it (dashed line in Fig. 11). The search direction is given by the leak probability of nodes in the vicinity of the one with highest probability.

\section{Discussion}

Leakage is detected in 8 out of the 9 faulty scenarios, as seen in Tab. 3. The 1 $\mathrm{l} / \mathrm{s}$ leak located in demand component $\mathbf{c}_{4}$ (S9) is the only one that has not been detected. The high consumption of the component $(\approx 30 \%$ of the total) masks the effect of the already low leakage water discharge (2.5\% distributed among all components) and consequently, the changes in detection indicators are not large enough to identify a leak.

The non-faulty scenario is tested by considering a validation scenario (S0) with different boundary conditions than the one used to set the thresholds. A good result is obtained as no false alarms are triggered during this scenario.

All the evaluated leaks have been located in the component with highest memberships in the leak zone. Memberships are defined depending on the nodes' pressure sensitivity, thus any anomaly that affects pressure will have a greater impact on the predominant demand component of the anomalous zone than in any other demand component. This was the expected behavior that motivated the use of geographically distributed parameters to locate leaks.

Detection times depend on the relation between leak size and water consumption of the predominant demand component in the leak zone. This relation is directly linked to the variations in calibrated demand components: low consumption demand components are more affected by leaks than high consumption ones, in the same way that leaks with high water discharge have a greater effect than leaks with low water discharge. Hence, large variations in demand components are instantly identified by the detection indicators, whereas small variations require a larger number of time samples to be analyzed to identify if an anomaly is occurring or not.

The leak membership method presents better results in terms of localization accuracy because it considers the effect of the leak on all demand components, 

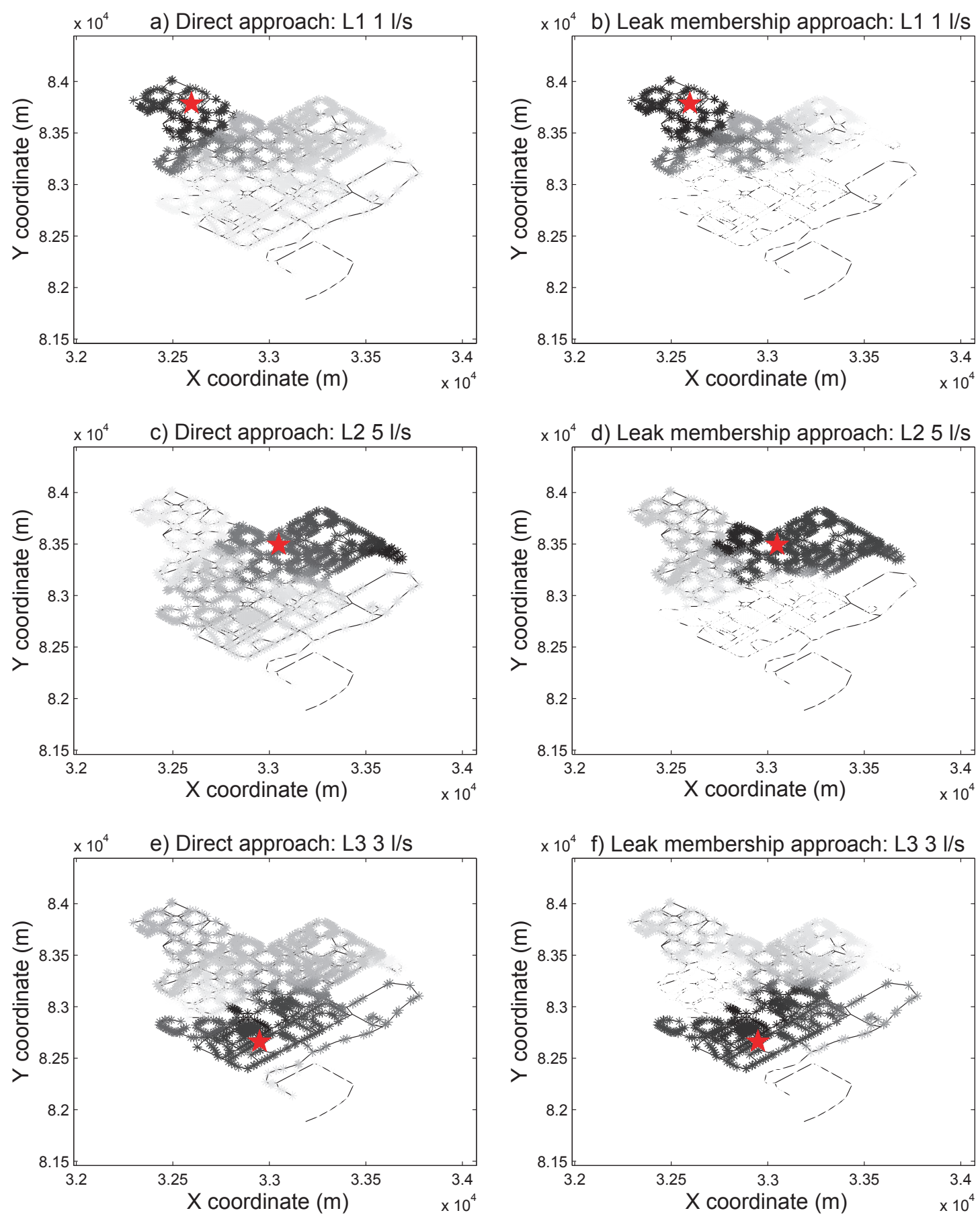

FIG. 9. Localization results for scenarios S3, S4 and S8 (rows) 
a) Direct approach: L1 $1 \mathrm{l} / \mathrm{s}$

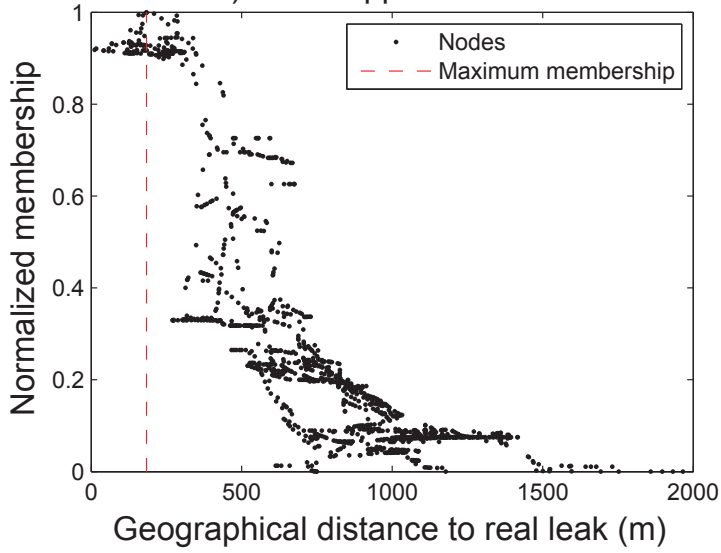

c) Direct approach: L2 $5 \mathrm{l} / \mathrm{s}$

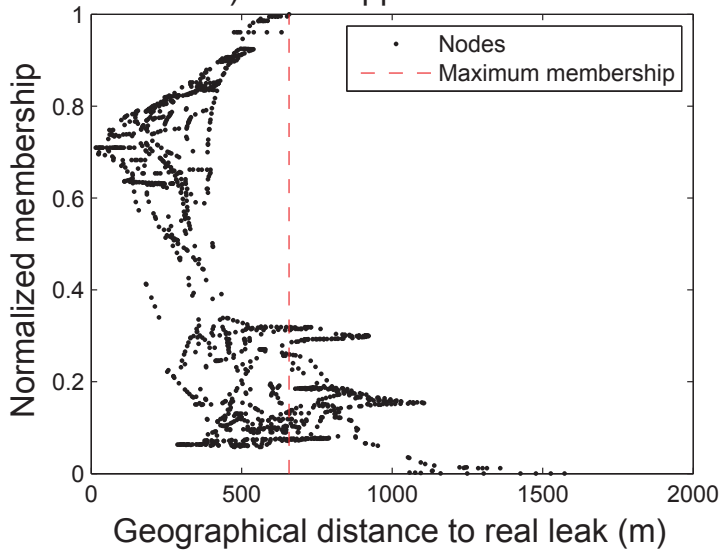

e) Direct approach: L3 3 I/s

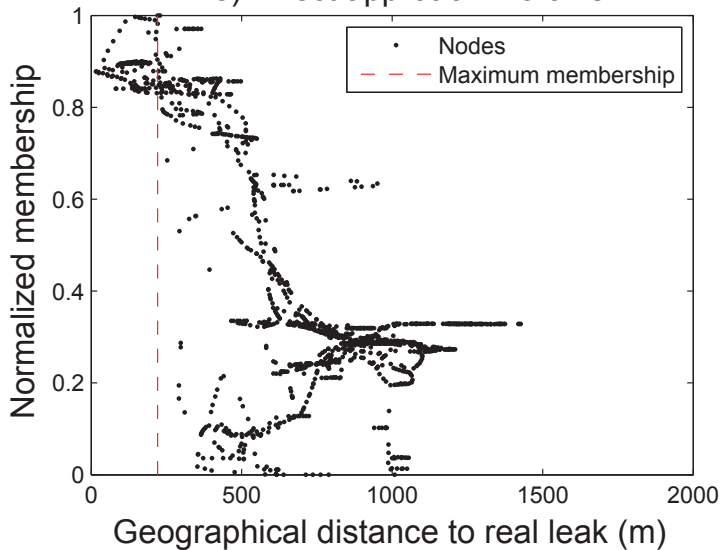

b) Leak membership approach: L1 $1 \mathrm{I} /$

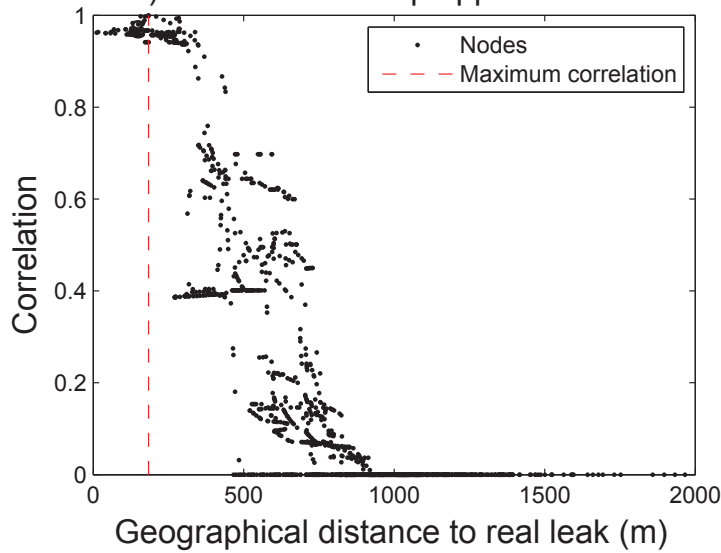

d) Leak membership approach: L2 5 I/

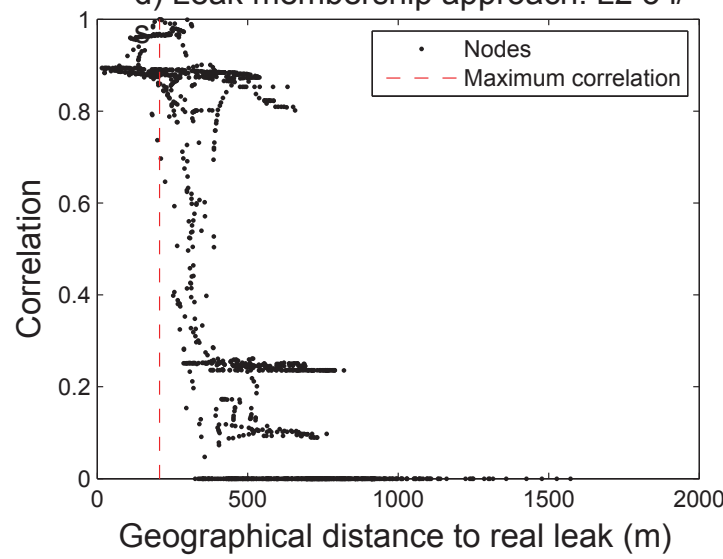

f) Leak membership approach: L3 3 I/s

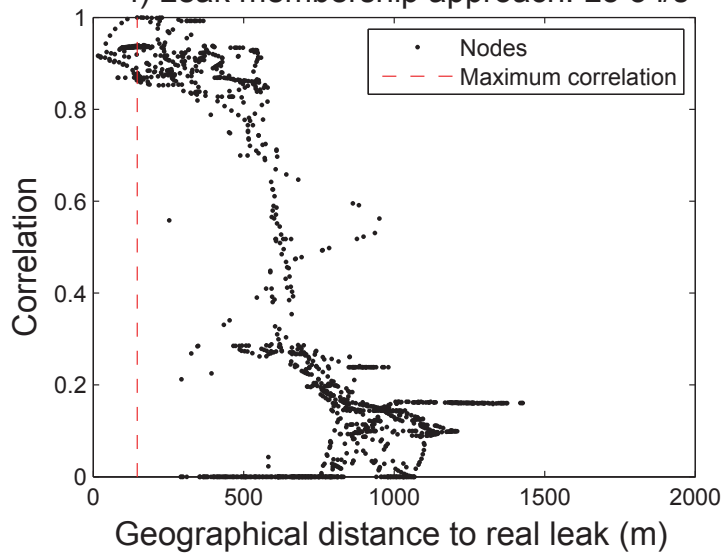

FIG. 10. Geographical distance from each node to the real leak depending on membership and correlation for scenarios S3, S4 and S8 (rows) 
a) Direct approach: L1 $1 \mathrm{l} / \mathrm{s}$

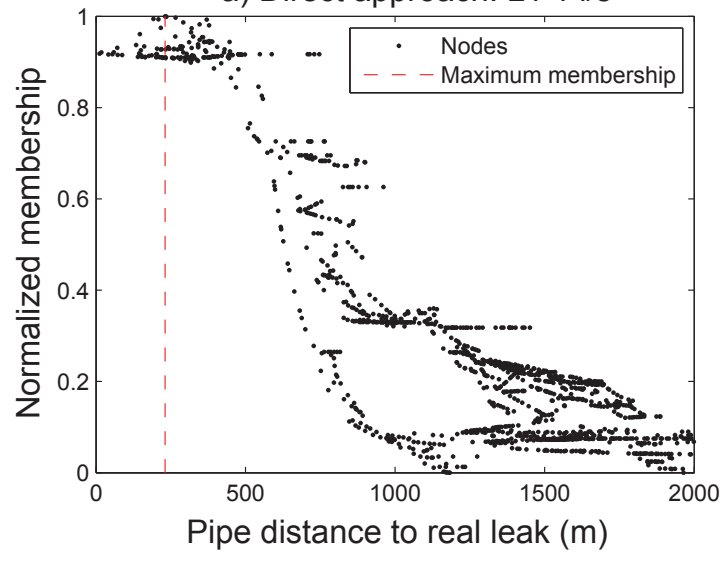

c) Direct approach: L2 $5 \mathrm{l} / \mathrm{s}$

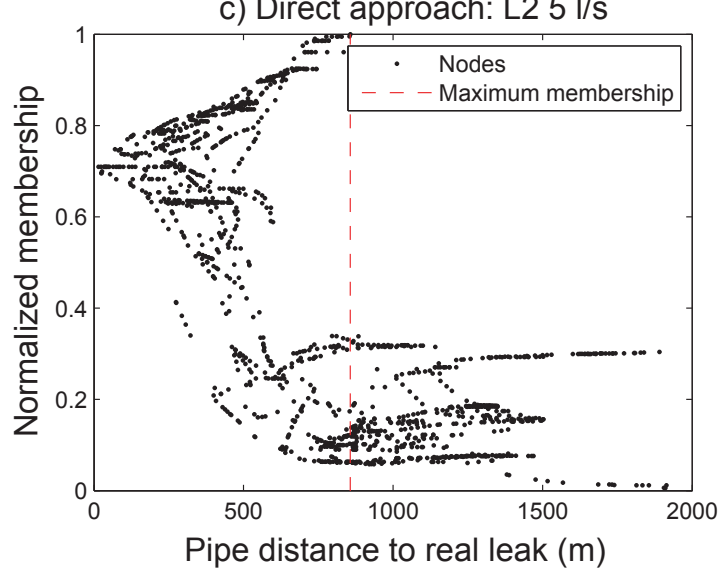

e) Direct approach: L3 3 l/s

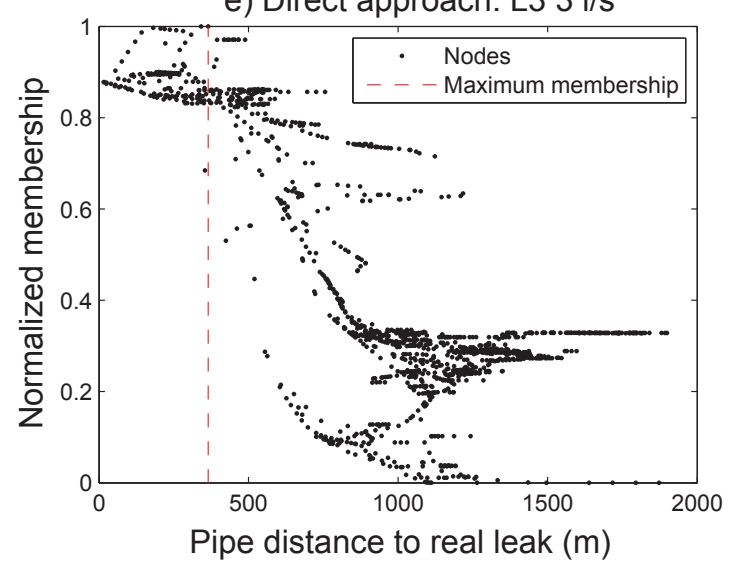

b) Leak membership approach: L1 1 l/s

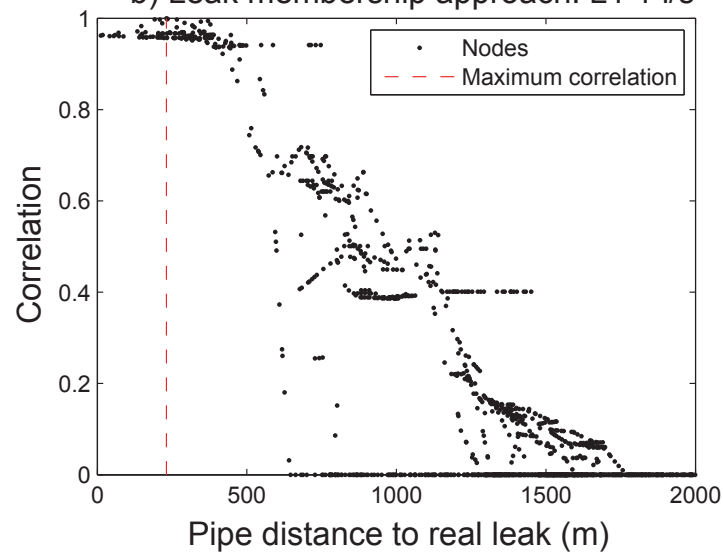

d) Leak membership approach: L2 5 l/s
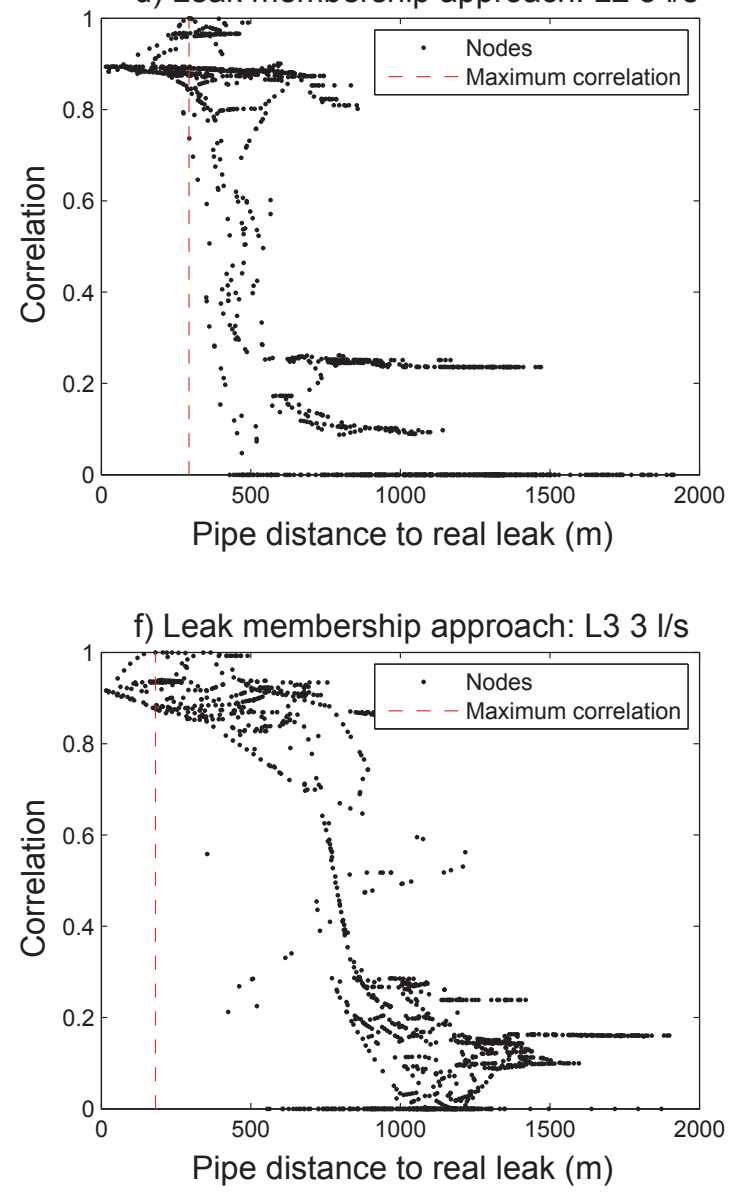

FIG. 11. Pipe distance from each node to the real leak depending on membership and correlation for scenarios S3, S4 and S8 (rows) 
whereas the direct method only considers the effect of the leak on the demand component with higher nodes' memberships in the leak zone. The localization accuracy generated by the leak membership method is about 180 meters in all scenarios except in case of S6. Pipe distances are greater than the geographic ones, but present an equivalent qualitative behavior in terms of accuracy, as seen in Fig. 10 and Fig. 11. The worst result is obtained for the $1 \mathrm{l} / \mathrm{s}$ leak 2 (S6) due to the small leak size together with its location in a zone where the predominant component has low memberships (30\%-40\%). The changes in the demand components are significant enough to detect the leak but not to locate it accurately.

The methodology is able to distinguish between demand evolution and burst appearance. Daily, weekly and seasonal changes cannot be confused with leakage because: 1) calibrated demands are considered to have daily periodicity; and 2) the comparison between demand components uses data from the same samples of the previous week. On the other hand, the long term evolution is progressively incorporated in the model by the continuous update of online calibrated demand components. This evolution is assumed to have slower impact on the online calibration than the one caused by a burst.

\section{CONCLUSIONS}

This work presents a leak detection and localization methodology combined with calibration. Leakage detection is based on the comparison between currently calibrated components and historical ones. Then, the geographical distribution of demand parameters allows a straightforward localization of the leak.

The methodology presented is a first step towards the integration of model calibration and leakage detection and location. In future stages the methodology can be modified to work with evolutionary methods so that the changes in demand components can be detected and classified (e.g. using ANNs) to detect and locate leakages or other anomalies; or the calibration methodology be based on GAs. Currently, the calibration methodology is LS-based and the detection and localization is based on the detection indicators analyses.

Detectability of leaks depends on the relation between the leak water discharge and demand components' consumption. Small leakages located in zones with high consumption components are not detectable due to the small variations caused on them.

Two methods are proposed to locate the leak in a specific area of the network. The leak membership method shows better accuracy in most of the tested scenarios as it considers the effect of the leak on all components. The method loses accuracy when considering small leaks $(1 \mathrm{l} / \mathrm{s})$ whose effect is distributed among several demand components.

In conclusion, leaks with a water discharge smaller than the affected components' uncertainty may be overlooked; or detected but located with low accuracy. This limitation can be improved by the inclusion of extra sensors that reduce the calibrated components' uncertainty. A second possible solution is to utilize 
these new sensors to increase the number of components, which would have less consumption and consequently, would be more sensitive to leakage. Additionally, leaks that induce pressure variations lower than sensors' uncertainty cannot be detected.

Apart from leaks, the method presented will detect unusual flows such as filling a tanker truck, fire flow tests, increased production at an industrial facility, special events or new large users coming on line. However, most of these unusual flows are short duration events. Hence, the methodology would detect two changes (increase and decrease) in a short period of time, differentiating from leaks that remain in the network until they are repaired. Other special events or new large users coming on line would be detected initially as a leak, but would be reclassified following the inspection by the water utility. Furthermore, the method can also be used to identify abrupt decreases in water use (e.g. school vacation, repair of a large leak, etc.).

The estimation of base demands is a key factor in the validity of the method presented. The increased use of Automated Meter Reading may make this method more feasible, i.e., providing more accurate base demands.

This paper presents a first analysis of a detection and localization method with promising results. However, the developed methodology has to be further tested in additional case studies under multiple conditions to be able to generalize the findings. Additional scenarios including multiple leaks will be analyzed to determine the ability to detect simultaneous burst. Future work will consider the minimum detectable leakage depending on sensors' resolution. Additionally, flow sensors will be tested and compared with pressure sensors in order to assess

which is the best option. A future real case test will be performed when having real data available. Finally, the comparison with other methods will be done to assess the applicability over other approaches.

\section{ACKNOWLEDGEMENTS}

This work was supported in part by the project FP7 - ICT - 2012 - 318556 (EFFINET) and FP7 - ICT - 2012 - 318272 (iWIDGET) of the European Commission, by the Project DPI2014 - 58104 - R (HARCRICS) and by the Polytechnic University of Catalonia. The model of the real network was provided by the Barcelona Water Company AGBAR. 


\section{REFERENCES}

ADEC (2000). "Technical Review of Leak Detection Technologies - vol.1 - Crude Oil Transmission Pipelines." Alaskan Department of Environmental Conservation, Alaska.

Colombo, A. F., Lee, P., and Karney, B. W. (2009). "A selective literature review of transient-based leak detection methods." Journal of Hydro-environment Research, 2(4), 212-227.

Farley, B., Mounce, S. R., and Boxall, J. B. (2011). "Field Validation of "Optimal" Instrumentation Methodology for Burst/Leak Detection and Location." Water Distribution Systems Analysis 2010, Reston, American Society of Civil Engineers, 1093-1102.

Giustolisi, O. and Walski, T. (2012). "Demand Components in Water Distribution Network Analysis." Journal of Water Resources Planning and Management, 138(4), 356-367.

Goulet, J.-A., Coutu, S., and Smith, I. F. (2013). "Model falsification diagnosis and sensor placement for leak detection in pressurized pipe networks." Advanced Engineering Informatics, 27(2), 261-269.

Henault, J.-M., Moreau, G., Blairon, S., Salin, J., Courivaud, J.-R., Taillade, F., Merliot, E., Dubois, J.-P., Bertrand, J., Buschaert, S., Mayer, S., and Delepine-Lesoille, S. (2010). "Truly Distributed Optical Fiber Sensors for Structural Health Monitoring: From the Telecommunication Optical Fiber Drawling Tower to Water Leakage Detection in Dikes and Concrete Structure Strain Monitoring." Advances in Civil Engineering, 2010, 1-13.

Hugenschmidt, J. and Kalogeropoulos, A. (2009). "The inspection of retaining walls using GPR." Journal of Applied Geophysics, 67(4), 335-344.

Hutton, C. J., Kapelan, Z., Vamvakeridou-Lyroudia, L., and Savić, D. A. (2014). "Dealing with Uncertainty in Water Distribution System Models: A Framework for Real-Time Modeling and Data Assimilation." Journal of Water Resources Planning and Management, 140(2), 169-183.

Kang, D. and Lansey, K. (2011). "Demand and Roughness Estimation in Water Distribution Systems." Journal of Water Resources Planning and Management, 137(1), 20-30.

Kapelan, Z., Savic, D., Walters, G., Covas, D., Graham, I., and Maksimovic, C. (2003). "An assessment of the application of inverse transient analysis for leak detection: Part I." International Conference on Advances in Water Supply Management, London.

Kim, S. H. (2005). "Extensive Development of Leak Detection Algorithm by Impulse Response Method." Journal of Hydraulic Engineering, 131(3), 201208.

Lansey, K., El-Shorbagy, W., Ahmed, I., Araujo, J., and Haan, C. (2001). "Calibration Assessment and Data Collection for Water Distribution Networks." Journal of Hydraulic Engineering, 127(4), 270-279.

Liggett, J. and Chen, L. (1994). "Inverse Transient Analysis in Pipe Networks." Journal of Hydraulic Engineering, 120(8), 934-955. 
Maier, H., Kapelan, Z., Kasprzyk, J., Kollat, J., Matott, L., Cunha, M., Dandy, G., Gibbs, M., Keedwell, E., Marchi, A., Ostfeld, A., Savic, D., Solomatine, D., Vrugt, J., Zecchin, A., Minsker, B., Barbour, E., Kuczera, G., Pasha, F., Castelletti, A., Giuliani, M., and Reed, P. (2014). "Evolutionary algorithms and other metaheuristics in water resources: Current status, research challenges and future directions." Environmental Modelling $\&$ Software, 62, 271-299.

Mounce, S. R., Boxall, J. B., and Machell, J. (2010). "Development and Verification of an Online Artificial Intelligence System for Detection of Bursts and Other Abnormal Flows." Journal of Water Resources Planning and Management, 136(3), 309-318.

Mounce, S. R., Mounce, R. B., and Boxall, J. B. (2011). "Novelty detection for time series data analysis in water distribution systems using support vector machines." Journal of Hydroinformatics, 13(4), 672.

Palau, C. V., Arregui, F. J., and Carlos, M. (2012). "Burst Detection in Water Networks Using Principal Component Analysis." Journal of Water Resources Planning and Management, 138(1), 47-54.

Pandya, P. and Gupta, V. (2014). "Enhancing Analog to Digital Converter Resolution Using Oversampling Technique." Blue Eyes Intelligence Engineering \&6 Sciences Publication Pvt. Ltd., 2(5).

Pérez, R., Puig, V., Pascual, J., Quevedo, J., Landeros, E., and Peralta, A. (2011). "Methodology for leakage isolation using pressure sensitivity analysis in water distribution networks." Control Engineering Practice, 19(10), 1157-1167.

Pérez, R., Sanz, G., Puig, V., Quevedo, J., Cuguero Escofet, M. A., Nejjari, F., Meseguer, J., Cembrano, G., Mirats Tur, J. M., and Sarrate, R. (2014). "Leak Localization in Water Networks: A Model-Based Methodology Using Pressure Sensors Applied to a Real Network in Barcelona [Applications of Control]." IEEE Control Systems, 34(4), 24-36.

Pilcher, R. (2007). "Leak location and repair guidance notes and... the never ending war against leakage." Water Loss 2.

Puust, R., Kapelan, Z., Savic, D. a., and Koppel, T. (2010). "A review of methods for leakage management in pipe networks." Urban Water Journal, 7(1), 25-45.

Romano, M., Kapelan, Z., and Savić, D. A. (2013). "Geostatistical techniques for approximate location of pipe burst events in water distribution systems." Journal of Hydroinformatics, 15, 634-651.

Romano, M., Kapelan, Z., and Savić, D. A. (2014). "Automated Detection of Pipe Bursts and Other Events in Water Distribution Systems." Journal of Water Resources Planning and Management, 140(4), 457-467.

Rossman, L. (2000). EPANET 2 Users Manual. Water Supply and Water Resources Division, National Risk Management Research Laboratory.

Sanz, G. and Pérez, R. (2014). "Comparison of Demand Pattern Calibration in Water Distribution Network with Geographic and Non-Geographic Parameterization." 11th International Conference on HydroInformatics, New York.

Sanz, G. and Pérez, R. (2015). "Sensitivity Analysis for Sampling Design and Demand Calibration in Water Distribution Networks Using the Singular 
Value Decomposition." Journal of Water Resources Planning and Management, 04015020 .

Savic, D., Kapelan, Z., and Jonkergouw, P. (2009). "Quo vadis water distribution model calibration?." Urban Water Journal, 6(1), 3-22.

Sumer, D. and Lansey, K. (2009). "Effect of Uncertainty on Water Distribution System Model Design Decisions." Journal of Water Resources Planning and Management, 135(1), 38-47.

Vítkovský, J., Simpson, A., and Lambert, M. (2000). "Leak Detection and Calibration Using Transients and Genetic Algorithms." Journal of Water Resources Planning and Management, 126(4), 262-265.

Walski, T., Sage, P., and Wu, Z. (2014). "What Does it Take to Make Automated Calibration Find Closed Valves and Leaks?." World Environmental and Water Resources Congress 2014, 555-565.

Wu, Z. and Sage, P. (2006). "Water Loss Detection Via Genetic Algorithm Optimization-Based Model Calibration." ASCE 8th International Symposium on Water Distribution System Analysis, Cincinnati.

Wu, Z. Y., Sage, P., and Turtle, D. (2010). "Pressure-Dependent Leak Detection Model and Its Application to a District Water System.

Wu, Z. Y. and Song, Y. (2012). "Optimization Model for Identifying Unknown Valve Statuses and Settings." Water Distribution Systems Analysis 2012, Adelaide. 